\title{
Práctica administrativa-contable: vinculación teoría y práctica en la formación inicial de docentes*
}

\author{
Lorenzo Estrada - Carla Leticia Paz Delgadob
}

\begin{abstract}
Resumen: Este artículo se focaliza en los hallazgos de un proyecto de investigación cuyo propósito es develar cómo las prácticas externas contribuyen a la vinculación de la teoría y la práctica en la formación inicial de docentes. Además, identificar las percepciones y las valoraciones que los involucrados en ese proceso hacen sobre esta experiencia formativa. El método combina técnicas cuantitativas y cualitativas con la intención de comprender la realidad en estudio. Se consideró la participación de estudiantes de la modalidad a distancia, docentes asesores y tutores de los centros de práctica. Los resultados de investigación evidencian que la implementación de esta experiencia formativa ha contribuido, en gran medida, a fortalecer y afianzar capacidades del campo disciplinar, así como el desarrollo de competencias genéricas.
\end{abstract}

Palabras clave: vinculación teoría y práctica; formación inicial de docentes; educación a distancia; prácticas externas; formación por competencias.

Recibido: 08 enero 2019 - Evaluado: 27 marzo 2019 - Aceptado: 26 abril 2019

* Este proyecto de investigación ha sido patrocinado por el Fondo de Apoyo a la Investigación de la UPNFM. This research project has been sponsored by the Research Funds-UPNFM. Este projeto de pesquisa foi patrocinada pelo Fondo de Apoyo a la Investigación da UPNFM.

a Universidad Pedagógica Nacional Francisco Morazán, Tegucigalpa, Honduras. E-mail: lestrada@upnfm.edu.hn

b Universidad Pedagógica Nacional Francisco Morazán, Tegucigalpa, Honduras. E-mail: cpaz@upnfm.edu.hn 


\title{
Accounting Administrative Practice: Linking Theory and Practice in Initial Teacher Training
}

\begin{abstract}
This article focuses on the findings of a research project whose purpose is to reveal how external practices contribute to linking theory and practice in initial teacher training and to identify the perceptions and assessments of participants about this training experience. The method combines quantitative and qualitative techniques in order to understand the reality studied. Distance students, advising teachers, and tutors of practice centers participated in the study. Research results show that the implementation of this training experience has helped, to a large extent, to strengthen and consolidate the capacities of this disciplinary field and to develop generic skills.
\end{abstract}

Keywords: Theory-practice relationship; initial teacher training; long distance education; external practices; competency training.

\section{Estágio administrativo-contábil: vinculação entre teoria e prática na formação inicial de docentes}

Resumo: Este artigo foca nos achados de um projeto de pesquisa cujo objetivo é demonstrar como os estágios contribuíram para vincular a teoria e a prática na formação inicial de docentes. Além disso, identificar as percepções e as considerações que os envolvidos nesse processo fazem sobre essa experiência formativa. O método combina técnicas quantitativas e qualitativas com a intenção de compreender a realidade em estudo. Foi considerada a participação de estudantes da modalidade a distância, docentes assistentes e tutores dos centros de estágio. Os resultados evidenciam que a implantação dessa experiência formativa contribui, em grande medida, para fortalecer e consolidar capacidades do campo disciplinar, além de desenvolver competências genéricas.

Palavras-chave: vinculação entre teoria e prática; formação inicial de docentes; educação a distância; estágios; formação por competências. 


\section{Introducción}

Los profesores se consideran un elemento primordial para el desarrollo humano y el bienestar social de una nación. Por tal razón, se ha instaurado a nivel global una tendencia encaminada a fortalecer la formación profesional docente. La etapa de preparación inicial es, sin lugar a dudas, de gran importancia, pues implica la construcción de la identidad profesional, el aprendizaje colaborativo, el dominio de la disciplina y su didáctica, así como la evaluación de los aprendizajes y la atención a las diferencias de los estudiantes. A pesar de esto, los modelos, los planes y los programas curriculares, así como las instituciones formadoras, no han logrado los perfiles de egreso esperados de esta etapa formativa. Estudiosos en la materia son contundentes en afirmar que la problemática consiste en la selección de los estudiantes de magisterio, el conocimiento de la materia, la fragmentación de las áreas de conocimiento, el perfil de los formadores de formadores, la poca vinculación con la vida escolar y la separación entre teoría y práctica (Berliner, 2000; Tenti, 2007; Vaillant y Marcelo, 2015).

Desde esta mirada, este trabajo se focaliza en analizar dos aspectos sustantivos en la formación de docentes del área de Educación Comercial en la modalidad a distancia. En primer lugar, identificar las percepciones y las valoraciones que los actores involucrados en el proceso de práctica tienen sobre esta experiencia formativa como mecanismo de vinculación entre teoría y práctica. En segundo lugar, determinar la importancia de los saberes del campo disciplinar (contabilidad, administración y finanzas) para este profesional de la docencia. En relación con el primer aspecto, Freixa, Novella y Pérez (2012) explican cómo a fin de consolidar los conocimientos disciplinares se requiere de un proceso de vinculación con el campo profesional mediante el desarrollo de prácticas externas que traen consigo las siguientes ventajas: a) están en la estructura curricular del plan de estudios, b) son parte de un espacio formativo que contribuye con la integración del saber hacer en contexto, c) se cuenta con un programa y docentes asesores tanto de la universidad como del centro de práctica, y d) se establece un vínculo entre la universidad y los espacios de desempeño profesional.

Es clave mencionar que la investigación educativa ha demostrado que los procesos de práctica constituyen una parte integral del desarrollo de docentes efectivos (Gray, Wright y Pascoe, 2017). Sin embargo, para que la práctica sea una experiencia formativa exitosa es necesario proporcionar situaciones de aprendizaje apropiadas para preparar a los profesores principiantes acerca de las realidades de la enseñanza (Grudnoff, 2011; Ralf, Walker y Wimmer, 2008), pues la práctica puede ser estresante en relación con la carga de trabajo, la presión de ser evaluado y la falta de pertenencia al contexto en el cual se realiza (Badali, 2008; Caires, Almeida y Martins, 2010; Gray et al. 2017).

Estas y otras características de las prácticas han despertado el interés de los investigadores. Por ejemplo, Clayton, Smith y Dyment (2014) indagaron sobre los componentes esenciales de un proceso de práctica en la formación inicial de docentes, y concluyen que se hace necesario promover la autoconciencia, la reflexión guiada, el intercambio de experiencias y el fomento de una comunidad de aprendizaje fuerte y segura. Por su parte, Tilson, Sandretto y Pratt (2017) examinaron las creencias de los estudiantes en los ciclos de práctica e identificaron que tienen dificultades para movilizar sus conocimientos teóricos en los espacios de desempeño profesional. El estudio realizado por Almeyda-Hidalgo (2016) detalla que la línea de investigación sobre la práctica en la formación inicial de docentes ha considerado diversas dimensiones para su estudio: los enfoques de formación predominantes, la reflexión sobre el saber docente, las relaciones entre teoría y práctica, la organización y el peso curricular de los espacios formativos de práctica, así como las estrategias que se ponen a disposición para el feedback a los estudiantes. Sumado a esto, la literatura empírica sobre el componente de práctica en los programas de formación docente está repleta de discusiones sobre los muchos beneficios que aporta al propósito de preparar a los docentes en preservicio para las realidades de la enseñanza en un contexto real (Choy, Wong, Goh y Ling Low, 2014). 
Al considerar nuestro segundo aspecto de estudio, es necesario destacar que el dominio de las competencias disciplinares es importante, pues es lo que se enseñará. En este sentido, Del Pozo, Fernández-Lozano, Gonzáles-Ballesteros y De Juanas (2013) concluyen que se recibe un nivel de conocimiento muy elemental durante los años de preparación para el ejercicio docente. La enseñanza efectiva requiere que los maestros estén bien informados y capacitados en tres dominios de contenido: a) materia, b) contexto de instrucción, y c) estrategias de instrucción (Darling-Hammond y Bransford, 2007; Polkinghorne, 2015). De acuerdo con Grossman, Schoenfeld y Lee (2005, p. 201):

dentro de la formación docente, el conocimiento de la materia está enmarcado en el conocimiento pedagógico y se entiende como: la capacidad de anticipar y responder a los patrones típicos de comprensión de los estudiantes, así como la capacidad de crear múltiples ejemplos y representación de temas desafiantes que conforman el contenido de tal manera que sea accesible a una amplia gama de estudiantes.

En la formación de docentes de educación comercial las competencias disciplinares se satisfacen mediante cursos de contabilidad, finanzas, economía, mercadotecnia, administración, derecho mercantil, comunicación y tecnología de la información (National Association for Business Teacher Education, 2010). Algunos estudios han aportado evidencias que apoyan cómo la participación en procesos de práctica administrativa financiera y contable contribuyen al logro de los saberes disciplinares (Loibl y Fisher, 2013; Polkinghorne, 2015).

Al tomar en cuenta estas tendencias, el análisis que presentamos se basa en los hallazgos de un estudio sobre la pertinencia del espacio de prácticas externas en el campo administrativo y contable para la formación inicial de docentes de educación comercial en la modalidad a distancia. Esto en cuanto sea un instrumento que permita a los profesores en preservicio contar con experiencias reales a la hora de facilitar procesos de aprendizaje referidos al campo de las finanzas, la contabilidad y la administración. En este sentido, se plantearon los siguientes interrogantes: a) ¿cuáles son las valoraciones de los estudiantes acerca de la práctica administrativa contable en el proceso formativo?, b) ¿qué bondades y limitaciones identifican los docentes asesores sobre esta experiencia formativa? y c) ¿cuáles son las apreciaciones de los tutores sobre la práctica administrativa contable en la formación de un educador comercial?

Con este trabajo hacemos una contribución a la literatura que busca comprender la importancia de las prácticas externas en la formación de los estudiantes. Además, nuestro estudio considera los actores de la modalidad a distancia, quienes hasta ahora han recibido una menor atención, de manera que se contribuye así a llenar ese vacío de conocimiento en esta área.

El resto del artículo se organiza de la siguiente forma. La segunda sección presenta la fundamentación teórica centralizada en la formación inicial de los docentes de educación comercial y el papel que juegan las prácticas externas en el proceso; la tercera describe el espacio formativo de la práctica administrativa contable; la cuarta aborda el método de investigación; la quinta sección muestra los resultados; la sexta presenta la discusión, y en la séptima se presentan las conclusiones.

\section{Referentes conceptuales}

Los procesos de enseñanza-aprendizaje en las instituciones de educación superior han cambiado en el último siglo, caracterizándose por docentes que implementan prácticas educativas de alto impacto (Kug, 2008). Estrategias didácticas, como, por ejemplo, el aprendizaje-servicio, la investigación en pregrado, las prácticas externas y los proyectos colaborativos, han demostrado de manera consistente beneficios para los estudiantes universitarios (Vizenor, Souza y Jordan, 2017). Además, la implementación de modelos pedagógicos como el enfoque por competencias han contribuido a reducir la brecha entre el mundo laboral y la academia (Zapata-Callejas, 2015).

En el caso de la formación inicial de docentes en educación comercial estas estrategias son de vital importancia para el desarrollo de competencias con alto grado de complejidad. Crew y Bodenhamer (2009) identificaron las habilidades 
que necesitan estos estudiantes antes de ingresar a ejercer la profesión, las cuales son: a) la gestión de la clase, b) el trabajo con diversos estudiantes, c) las habilidades organizacionales, d) la planificación de la lección, e) la didáctica de la disciplina, y f) competencias genéricas o blandas que se adquieren en contextos reales de práctica profesional (Gonzáles y Larraín 2005).

\section{Formación docente en el campo de la educación comercial}

La educación comercial refiere a la promoción de espacios pedagógicos para el desarrollo de competencias profesionales orientadas a la gestión del apoyo administrativo y la gestión empresarial (Canales-García y Araya-Muñoz, 2017). Ese espacio profesional de la docencia se encarga de formar personal técnico-profesional en las especialidades de administración de oficinas, atención de centros de servicio al cliente y diversas tareas de apoyo logístico y administrativo. Entenderemos la educación comercial como un conjunto de procesos técnicos administrativos aplicados al ámbito de las organizaciones que se vinculan con las ofertas educativas a nivel secundario, relacionadas con las áreas de la administración, la contabilidad y las finanzas. Desde esa perspectiva, este ámbito de la educación aporta al desarrollo socioeconómico de un país, al contribuir en la formación empresarial y emprendedora de la población y propiciar espacios de inclusión financiera. Por tanto, la docencia en esta área tiene como enorme desafío la preparación de docentes con un alto grado de competencias en "la toma de decisiones propositivas y proactivas, con ética y valores, que no se constituyan en meros transmisores de contenidos sin mayor relación con los problemas sociales existentes" (Araya-Muñoz y España-Chavarría, 2012, p. 170).

\section{La práctica administrativa- contable como espacio de vinculación entre teoría y práctica}

Según Moreira, Vieira y Silva (2015) las universidades deben proporcionar una formación que trascienda la esfera de la dimensión teórica. Para esto, será necesario la construcción de un conocimiento emancipador que permita establecer el vínculo dialéctico entre la teoría y la práctica, con el propósito de formar profesionales capaces de desempeñar sus funciones en la realidad social en la que se encuentran inmersos. La investigación ha demostrado que existen aún muchos desafíos en la formación académico profesional. Uno de estos -quizás el más importante- consiste en superar la separación del conocimiento de su contexto histórico y social, y generar así una visión fragmentada de la realidad, ya que existe una desarticulación entre la teoría y la práctica, lo cual, al final, repercute en la falta de oportunidades para el aprendizaje reflexivo (Linuesa, 2007; Korthagen y Kessels, 2009; Rozada, 2007). A fin de superar la visión atomizada de la formación tradicional será necesario que la preparación de profesionales se acompañe de procesos de acercamiento y desempeño en contextos reales (Fletcher, Mountjoy y Bailey, 2011).

De acuerdo con esta perspectiva, los espacios de práctica en la formación académica del individuo constituyen un eslabón de indiscutible relevancia a fin de situarlo en un contexto en el que deberá ser capaz de desarrollar y aplicar destrezas, habilidades y conocimientos frente a un puesto de trabajo específico en el que debe mostrar lo que aprendió a lo largo de su formación, y establecer así una vinculación entre lo estudiado (teoría) y las funciones o actividades a desarrollar en la empresa (práctica). Sin embargo, su importancia no radica solo en la observancia de estos elementos; Von Feigenblatt (2015) concluye que la integración de la teoría y la práctica en la educación de negocios no solo es necesaria por razones funcionales, sino más bien es una necesidad en el propósito de fomentar la formación de líderes éticos y bien preparados.

Kane, Berryman, Goslin y Meltzer (1990) plantean que la práctica proporciona sentido y motivación para aprender, además de que ayuda a romper la separación entre el conocimiento (saber qué) y la práctica (saber cómo y cuándo). Según estos autores este contexto de aprendizaje se debe diseñar para: a) la integración de los conocimientos, los procedimientos y su aplicación, b) la participación de múltiples habilidades de diferentes tipos que requieran integrarlas en una actuación, 
y c) la inclusión de las interacciones sociales, los recursos y las herramientas típicas de situaciones del mundo laboral. Adicionalmente, la creación de estos espacios de práctica ofrece a los estudiantes la oportunidad de sumergirse en un ambiente de responsabilidad profesional al observar la articulación entre la teoría y la práctica en un contexto real de actividad laboral (Tejada-Fernández, Carvalho-Dias y Ruiz-Bueno, 2017).

Sin lugar a dudas, la formación académica es necesaria, pero si no trasciende al mundo profesional y laboral se obtiene como resultado una visión fragmentada de la realidad. Grosu, Almasan y Circa (2015) opinan que los profesionales cuentan con capacidades que provienen del mundo académico, pero algunas competencias solo podrán adquirirse si se guía a los estudiantes en los procesos de práctica profesional. Lo anterior tiene como fin identificar de manera adecuada las habilidades y los conocimientos del ejercicio de la profesión.

En síntesis, la institución universitaria debe construir los puentes entre teoría y práctica, ambos elementos formativos que deben ser un contínuum en la formación de los profesionales y corresponder de esta manera a las exigencias de la sociedad actual.

\section{La práctica administrativa contable en la modalidad a distancia de la Universidad Pedagógica Nacional Francisco Morazán (UPNFM)}

La educación a distancia surge como una respuesta a la demanda de educación por parte de las personas que, por distintos motivos, no pueden acceder a la educación presencial, sobre todo en el nivel superior (Chaves-Torres, 2017). En este sentido, la UPNFM ofrece, en su modalidad a distancia, la carrera de Educación Comercial como una opción para la población interesada en el estudio de dicha área. En cuanto es parte de la estructura curricular de esta licenciatura, los estudiantes deben aprobar el espacio formativo de Práctica Administrativa Contable (PAC).
La PAC es una experiencia que permite al estudiante enfrentarse a la realidad laboral en las diferentes empresas o instituciones públicas o privadas del país. En ella, el estudiante asume el rol de empleado, en específico en las áreas de administración, contabilidad, finanzas y auditoría, entre otras, relacionadas con la especialidad. La duración de esta práctica corresponde a un periodo académico en el que deberá cumplir un mínimo de cuatrocientas horas de ejecución, al asistir al centro de práctica en la jornada laboral que se le asigne. El proceso está organizado en tres etapas: 1) preparación, 2) ejecución y 3) evaluación.

La etapa de preparación consiste en el desarrollo de un seminario de inducción en el que se brindan los lineamientos generales y la normativa de la práctica, así como se abordan temas relacionados con el quehacer laboral. En la etapa de ejecución, el estudiante asume su papel de empleado y lo orienta y supervisa el tutor responsable en el lugar de trabajo (jefe inmediato); de igual forma, lo supervisa de manera regular el docente asesor de la universidad. Finalmente, la etapa de evaluación consiste en la presentación de rúbricas de evaluación por parte del tutor y el asesor de práctica. Además, el estudiante debe realizar un informe escrito y la presentación oral de su proceso de práctica.

Durante las tres etapas, el estudiante y el asesor de práctica se mantienen en constante comunicación y retroalimentación, principalmente, a través de dos vías. Primero, a través de la plataforma virtual (aula virtual), a la que todos los estudiantes tienen acceso (la educación a distancia funciona bajo la modalidad в-Learning). Segundo, durante el periodo académico es obligatorio el cumplimiento de al menos cinco encuentros de acompañamiento tutorial (presencial), en los que se realizan jornadas de intercambio de experiencias.

\section{Metodología}

\section{Tipo de investigación}

Como método de investigación se utilizó el estudio de casos, el cual consiste en una descripción y un análisis de un fenómeno específico y complejo (Yin, 2013). Otros autores, como, por ejemplo, 
Álvarez y Hevia (2013), han utilizado esta perspectiva metodológica con el fin de comprender las relaciones entre la teoría y la práctica en la formación inicial de docentes. El alcance es de tipo descriptivo-interpretativo pues se espera valorar, desde la perspectiva de los actores, los aportes, las bondades y las limitaciones de la práctica administrativa contable en la formación inicial de docentes de educación comercial.

\section{Contexto del estudio}

La investigación se desarrolló en el Centro Universitario de Educación a Distancia de la UPNFm de Honduras, específicamente en la carrera de Profesorado de Educación Comercial, la cual incluye en su estructura curricular la Práctica Administrativa Contable como un espacio de práctica externa encaminado a fortalecer las competencias disciplinares relacionadas con las finanzas, la administración, y la contabilidad.

\section{Participantes}

Se tomó en consideración la participación de tres grupos: a) 33 estudiantes del Sistema de Educación a Distancia de último año del Profesorado en Educación Comercial que se encontraban desarrollando el proceso de práctica; b) 4 docentes asesores de práctica, y c) tutores en los centros de práctica (estos son empresas comerciales, ONG, banca privada, cooperativas de ahorro y crédito, entre otros).

\section{Técnicas e instrumentación}

En el caso de los docentes asesores se utilizó la entrevista semiestructurada, caracterizada por desarrollarse a través de líneas temáticas o de una guía con interrogantes flexibles que pueden adaptarse a cada entrevistado (Cifuentes, 2011). En este sentido, se diseñó como instrumento un guion de preguntas abiertas para la producción de información, orientado a identificar las valoraciones del profesorado acerca de las bondades y limitaciones de la Práctica Administrativa Contable. Posteriormente, se evaluó su pertinencia a través de la revisión de criterios por parte de cuatro docentes especialistas del área de educación comercial. Al tomar en consideración las sugerencias de mejora por parte de los expertos, se ajustó el instrumento a fin de obtener su versión final.

Para realizar la consulta a los estudiantes se consideró la administración de un cuestionario. Se efectúo una búsqueda de instrumentos orientados a valorar los procesos de prácticas externas, pero no fue posible recuperar alguno que se ajustara al contexto. Debido a esto, se elaboró un cuestionario ad hoc al estudio. A continuación, un grupo de siete jueces especialistas de educación comercial, con una experiencia promedio de diez años en el área, analizó el instrumento para determinar su validez a nivel de contenido. Se utilizó el procedimiento propuesto por Lawshe (1975), quien sugiere que un IVC igual a 0,29 será adecuado cuando se hayan utilizado cuarenta expertos, un IVC igual 0,51 será suficiente con catorce expertos, pero un IVC de al menos 0,99 será necesario cuando el número de expertos sea de siete o inferior. Como producto de este proceso se eliminaron los ítems que obtuvieron puntuaciones inferiores a 0,99 . Una vez reestructurado el instrumento, el Índice de Validez de Contenido obtenido fue de 1 . El estudio piloto permitió definir la confiabilidad al emplear el estadístico alfa de Cronbach, cuyo resultado fue de $\alpha=$ 0,91 , considerado excelente ${ }^{1}$.

De acuerdo con los tutores, en el centro de práctica se optó por utilizar el cuestionario para empleadores de la Universidad de Chile. Este instrumento, en su versión original, cuenta con 29 ítems distribuidos en tres dimensiones. Tras la adaptación al contexto de esta investigación, el número de ítems se redujo a 20 y se reestructuró en cuatro secciones: a) datos de la organización, b) desempeño de capacidades específicas, c) satisfacción general y d) un componente de preguntas abiertas destinado a sugerencias y comentarios. Para estudiar las propiedades psicométricas y determinar la validez de contenido se utilizó el procedimiento de Lawshe (1975) aplicado al grupo de expertos que participaron en el análisis del

1 George y Mallery (2003, p. 231) sugieren los siguientes valores para evaluar el coeficiente alfa de Cronbach: $\alpha>0,9$ es excelente, $\alpha>0,8$ es bueno, $\alpha>0,7$ es aceptable, $\alpha>0,6$ es cuestionable, $\alpha$ $>0,5$ es Pobre y $a<0,5$ es inaceptable. 
instrumento de los estudiantes. Como resultado del proceso de validez se eliminaron los ítems que obtuvieron puntuaciones inferiores a las que sugiere el IVC para siete evaluadores, el cual es de 0,99 . Una vez reformado el cuestionario, el IVC fue igual a 1 , y su confiabilidad fue de $\alpha=0,85$. Esto indica que el cuestionario posee una buena consistencia interna de los ítems. En ambos cuestionarios se incluyeron preguntas abiertas con la finalidad de que los participantes estuvieran en capacidad de responder de forma libre y de detallar sus opiniones, actitudes y conocimientos sobre los cuestionamientos planteados en él (Abarca, Alpízar, Rojas y Sibaja, 2012).

\section{Plan de análisis de los datos}

A fin de analizar los cuestionarios de los tutores y de los estudiantes se utilizó el software IBM SPSs Statistics 23. Para analizar las entrevistas de los asesores de práctica se utilizó el software MAXQDA 12 , en consideración a los procedimientos de comparación constante y la triangulación como mecanismos de validación de nuestras interpretaciones. Cabe señalar que, para la información cualitativa - por ser de naturaleza interpretativa- se empleó el ciclo de tareas propuestas por Miles y Huberman (1984), el cual implica: a) la reducción de los datos (codificación), b) la disposición de los datos (generar categorías) y c) la extracción de resultados y la verificación de conclusiones que permitan explicar el fenómeno en estudio.

\section{Resultados}

El análisis se realizó a partir de los tres actores involucrados en la práctica. Por su parte, la construcción de las categorías de análisis ha seguido la lógica inductiva, de tal manera que los significados emergen en el proceso.

\section{Valoración de los estudiantes sobre las prácticas externas en el proceso formativo}

Desde la perspectiva de los estudiantes, el proceso de práctica asume dos grandes líneas. Una centrada en los beneficios y otra en las dificultades.
En la Figura 1 se esquematizan estas dos apreciaciones, de modo que se resalta en el nivel de los beneficios el acercamiento al mundo de las organizaciones, la aplicación de conocimientos teóricos y la oportunidad de potenciar las habilidades del trabajo en equipo. En cuanto a las dificultades sobresalen la gestión del tiempo, la carga académica, los factores económicos y el poco conocimiento de software. Es posible valorar que el espacio formativo de práctica externa en ambientes laborales orientados a la administración, la contabilidad y las finanzas contribuye a configurar un sistema de experiencias disciplinares fundamentales para un docente de educación comercial. Se trata de construir un cúmulo de situaciones laborales que sirvan a la hora de facilitar el aprendizaje del área comercial en los programas de enseñanza secundaria. De igual forma, los estudiantes indican que la práctica externa genera un espacio para aplicar y potenciar las competencias disciplinares del plan de estudios. Se valora la experiencia del trabajo en equipo y que, al ser parte de una organización, deben actuar de forma colaborativa para solventar situaciones del entorno laboral. Esta mirada puede identificarse en la siguiente reflexión: "La práctica administrativa contable me ha enriquecido, pues he logrado tener contacto con un ambiente real del área comercial, yo he logrado contrastar mis conocimientos e información recibida en la universidad con la práctica en un espacio real" (Estudiante 23, comunicación personal, 30 de abril de 2016).

$\mathrm{Al}$ considerar las dificultades es necesario destacar que el aspecto económico representa una situación adversa para los estudiantes, pues deben abandonar su trabajo para cumplir con el tiempo reglamentado de práctica. La gestión del tiempo es otro elemento que se destaca, dado que el perfil de los estudiantes, en su mayoría, corresponde a personas con responsabilidades familiares y económicas, y estas condiciones limitan la experiencia en el entorno laboral. Para finalizar, los participantes refieren tener dificultades con el dominio de software contable y el uso de Excel, pues difiere de lo aprendido en la universidad y se requiere de tiempo y esfuerzo para apropiarse de este nuevo conocimiento. 


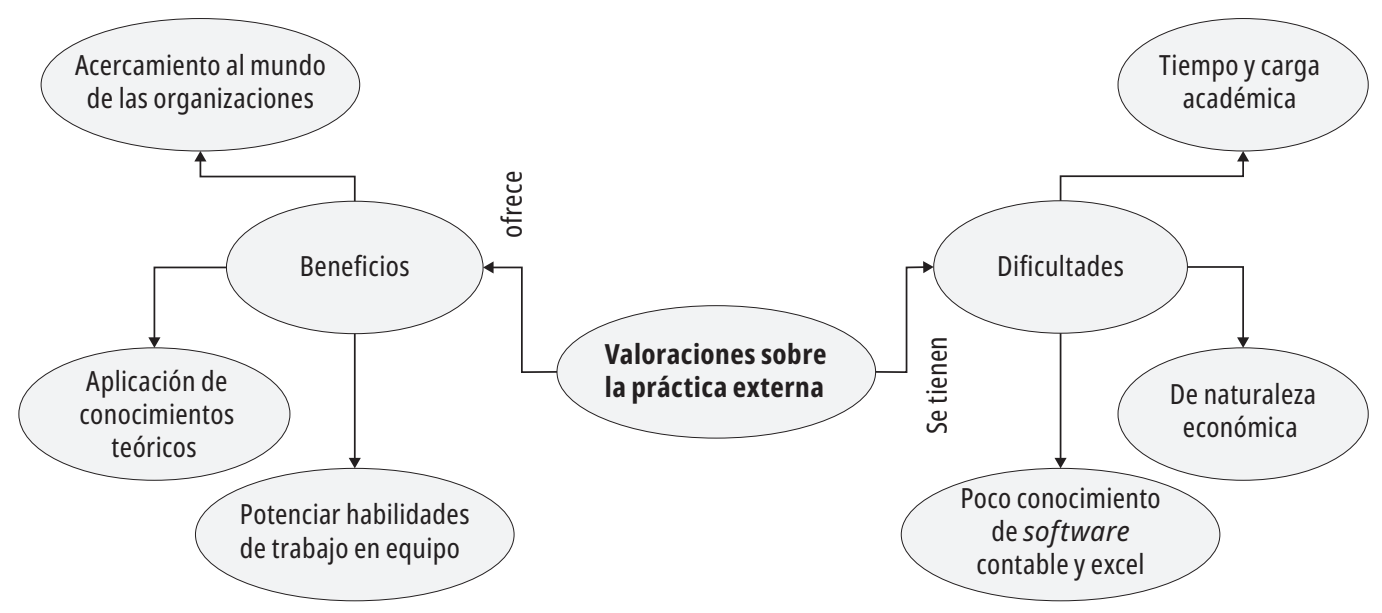

Figura 1. Valoraciones de los estudiantes sobre las prácticas externas. Fuente: elaboración propia a partir del análisis de la información.

Otro aspecto relevante es el desarrollo de las competencias disciplinares. La Tabla 1 muestra la autoevaluación de los estudiantes sobre el desarrollo de estas a lo largo de su formación y su fortalecimiento durante el desarrollo de la Práctica Administrativa Contable. La primera columna contiene las competencias específicas que el estudiante debe lograr al concluir el proceso de práctica; la segunda indica la media obtenida por cada aspecto; la tercera señala la desviación estándar, $y$, finalmente, se incluye la frecuencia y el porcentaje obtenido para cada uno de los elementos de la escala. En general, el grupo de competencias evaluadas puntuó por arriba de la media, lo que indica que los estudiantes consideran tener un nivel medio de desempeño en los saberes disciplinares, y son los más altos; el registro de las operaciones mercantiles $(\boldsymbol{\mu}=2,67)$, realizar el cierre contable de una empresa $(\boldsymbol{\mu}=2,52)$, y resolver problemas relacionados con la actividad financiera $(\mu=2,39)$.

Tabla 1. Nivel de logro de las competencias específicas

\begin{tabular}{|c|c|c|c|c|c|c|c|c|c|c|}
\hline \multirow{2}{*}{ Competencias } & \multirow[b]{2}{*}{$\boldsymbol{\mu}$} & \multirow[b]{2}{*}{$\boldsymbol{\sigma}$} & \multicolumn{2}{|c|}{ Nada } & \multicolumn{2}{|c|}{ Poco } & \multicolumn{2}{|c|}{ Suficiente } & \multicolumn{2}{|c|}{ Mucho } \\
\hline & & & $f$ & $\%$ & $f$ & $\%$ & $f$ & $\%$ & $f$ & $\%$ \\
\hline 1. Registrar operaciones mercantiles. & 20,67 & 00,48 & - & - & - & - & 11 & 330,3 & 22 & 660,7 \\
\hline 2. Registrar contablemente los elementos del costo. & 20,27 & 00,67 & 1 & 30,0 & 1 & 3,0 & 19 & 570,6 & 12 & 360,4 \\
\hline 3. Aplicar la legislación nacional en materia mercantil. & 20,36 & 00,65 & - & - & 3 & 90,1 & 15 & 450,5 & 15 & 450,5 \\
\hline 4. Realizar el cierre contable de una empresa. & 20,52 & 00,67 & - & - & 3 & 90,1 & 10 & 300,3 & 20 & 600,6 \\
\hline $\begin{array}{l}\text { 5. Elaborar estados financieros de acuerdo a las Normas } \\
\text { Internacionales de Información Financiera. }\end{array}$ & 20,12 & 00,89 & 1 & 30,0 & 8 & 240,2 & 10 & 300,3 & 14 & 420,5 \\
\hline $\begin{array}{l}\text { 6. Aplicar las tasas vigentes para el cálculo de los } \\
\text { diferentes impuestos. }\end{array}$ & 20,36 & 00,78 & - & - & 6 & 180,2 & 9 & 270,3 & 18 & 540,5 \\
\hline $\begin{array}{l}\text { 7. Resolver problemas relacionados con la actividad } \\
\text { financiera. }\end{array}$ & 20,39 & 00,56 & - & - & 1 & 30,0 & 18 & 540,5 & 14 & 420,5 \\
\hline $\begin{array}{l}\text { 8. Utilizar el software para resolver ejercicios prácticos } \\
\text { de contabilidad, matemática y administración. }\end{array}$ & 20,21 & 00,74 & - & - & 6 & 180,2 & 14 & 420,4 & 13 & 390,4 \\
\hline $\begin{array}{l}\text { 9. Interpretar documentos de carácter administrativo y } \\
\text { financiero. }\end{array}$ & 20,06 & 00,79 & 1 & 30,0 & 6 & 180,2 & 16 & 480,5 & 10 & 300,3 \\
\hline 10. Utilizar los conceptos de matemática financiera. & 20,30 & 00,64 & - & - & 3 & 90,1 & 17 & 510,5 & 13 & 390,4 \\
\hline
\end{tabular}

Fuente: elaboración propia. 


\section{Opinión de los docentes asesores sobre las bondades y las limitaciones de las prácticas externas}

Del proceso de codificación de las entrevistas a los docentes asesores emergieron dos categorías. Una referida a las bondades de la experiencia de práctica y la otra referida a sus limitaciones. Para las interpretaciones derivadas del proceso de triangulación se diseñó un mapa que detalla las tendencias finales (véase la Figura 2). Este mapa presenta aquellos elementos que, según los estudiantes, se fortalecen con la realización de la práctica; además, muestra los factores que representan un obstáculo para su ejecución. En este sentido, a nivel de las bondades se interpreta que el contacto con el mundo laboral favorece el desarrollo de competencias blandas tales como los procesos asertivos de comunicación, el desarrollo de la autoconfianza y la capacidad de trabajar cooperativamente en un equipo. Los asesores consideran que la experiencia de práctica fortalece la autoestima y les permite corroborar su potencial de aprendizaje.
Otro de los beneficios radica en la formación académica, pues la práctica ofrece un espacio contextualizado entre los conocimientos teóricos y los prácticos, los cuales deben conjugarse en el ejercicio de funciones propias del campo ocupacional. Las prácticas son una vía para desarrollar niveles complejos de competencias genéricas y específicas del educador comercial, y, sobre todo, generan una experiencia laboral real que consolida los conocimientos académicos. Además, los estudiantes adquieren experiencias que le son útiles a la hora de implementar la práctica profesional docente, ya que pueden utilizar algunos ejemplos o anécdotas de lo que les ocurrió en el proceso de enseñanza con los estudiantes de los institutos de educación media:

los estudiantes comentan que tienen mayor autoridad de conocimiento cuando imparten un espacio en el cual ellos han tenido una vivencia real en las practicas, es más, van a los centros educativos con un porte profesional diferente. Cuando se trata de hacer informes, planes, proyectos, ellos resaltan entre los demás estudiantes, fortalecen sus competencias docentes y realmente si nos ubicamos

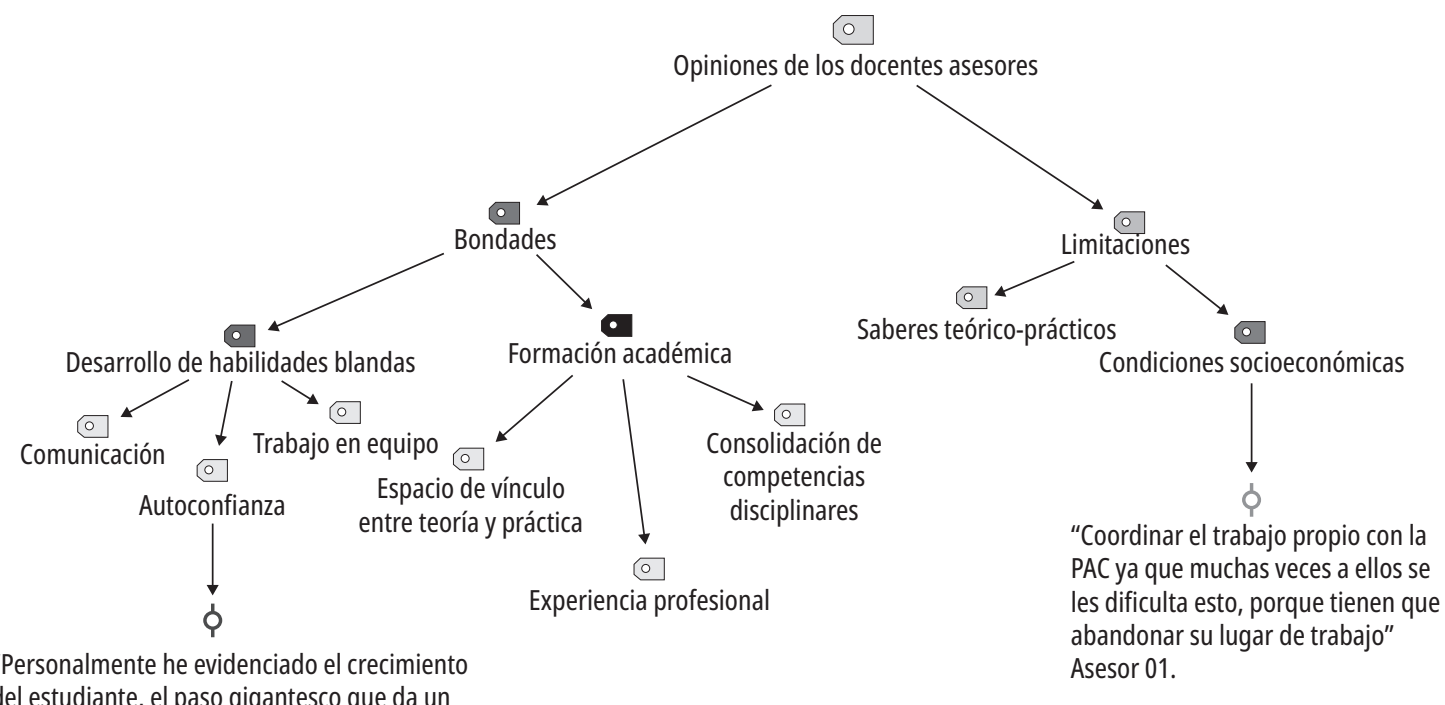

del estudiante, el paso gigantesco que da un estudiante de la Carrera de Educación Comercial después de su Práctica Administrativa Contable; no solamente el crecimiento profesional, si no el crecimiento como persona, como individuo y también empoderado a sus competencias" Asesora 04.

Figura 2. Opiniones de los asesores de práctica. Fuente: elaboración propia a partir del análisis de la información. 
en la misión de la universidad es formar cuadros docentes, entonces la práctica administrativa contable aporta en mucho. (Asesora 03, comunicación personal, 5 de mayo de 2016)

Las limitaciones identificadas son de naturaleza socioeconómica, aspecto que coincide con las valoraciones de los estudiantes. Se ratifica el hecho de que muchos deben renunciar a su puesto de trabajo, y para otros - cuyo ingreso familiar es bajo- el desarrollo de la práctica es una situación desafiante. Por otra parte, los asesores perciben que algunos estudiantes muestran dificultades para establecer conexiones entre los saberes teóricos y prácticos, así como su aplicación en el contexto laboral. Para dar respuesta a esta situación, los docentes efectúan acciones de orientación y reflexión en los espacios destinados a la asesoría académica.

\section{Apreciaciones de los tutores sobre la práctica en la formación del educador comercial}

Conocer y comprender las apreciaciones de los tutores en los centros de la práctica administrativa y contable ofrece la mirada de un actor sustantivo de la experiencia, pues es el profesional que acompaña de forma permanente a los estudiantes. En el análisis de la información se configuraron tres categorías que se presentan en la Figura 3. Este esquema ilustra las tendencias obtenidas del proceso de análisis textual que consisten en aspectos relativos al desempeño profesional de los estudiantes, presenta aquellas situaciones que pueden contribuir a una mejor experiencia formativa $y$, finalmente, muestra los aportes de la práctica a la vida profesional.

Al efectuar el análisis a partir de las categorías encontramos que, con respecto al fortalecimiento de competencias específicas de los estudiantes, los tutores opinan que los estudiantes cuentan con conocimientos teóricos y prácticos del ámbito empresarial, pero se requiere fortalecer capacidades tales como el uso de software contable, aspectos de auditoría, emprendedurismo y la aplicación de las Normas Internacionales de Información Financiera.

A partir de esta situación surge la categoría "sugerencias para la mejora de la práctica", desde la cual los tutores proponen incrementar el tiempo de práctica y fortalecer los vínculos con los docentes asesores de la universidad a fin de mejorar los procesos de retroalimentación y consolidar estas capacidades. Finalmente, los asesores concuerdan

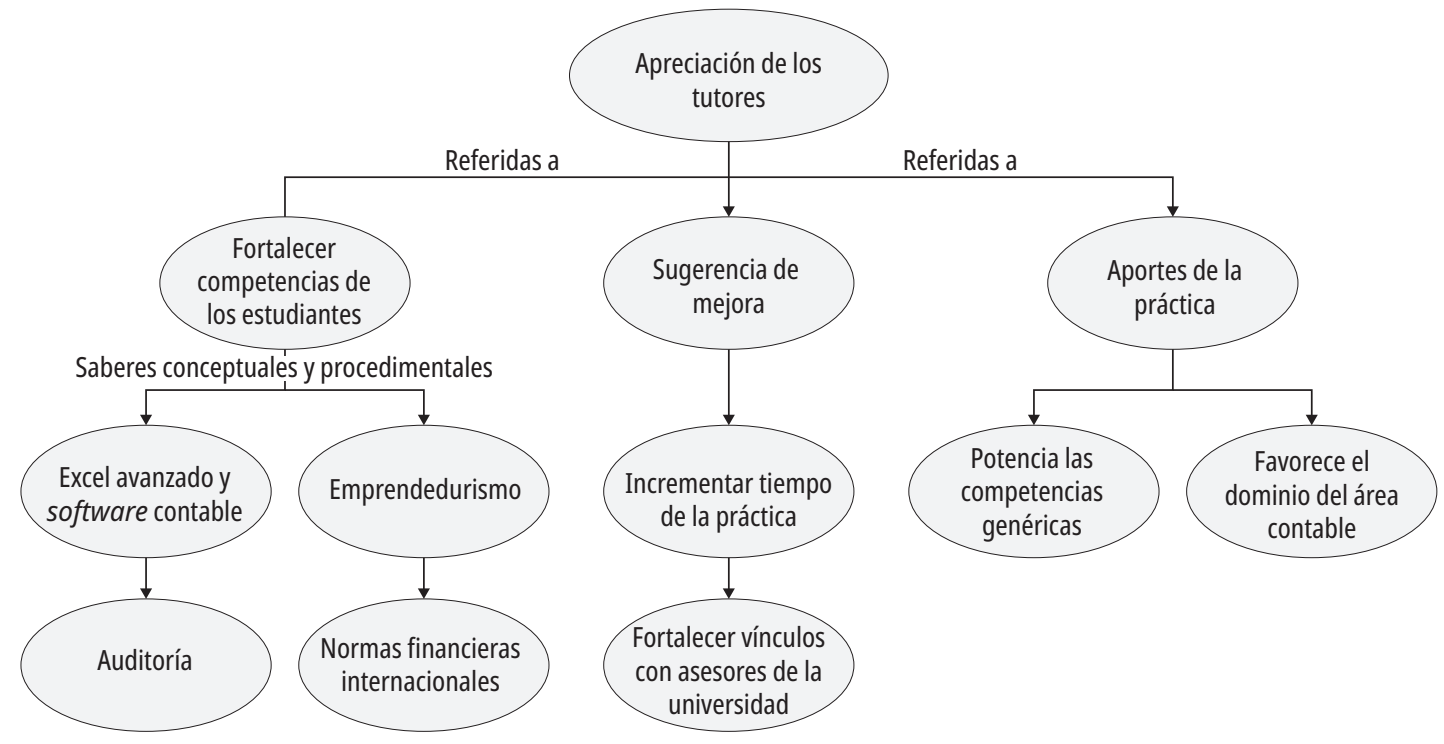

Figura 3. Apreciaciones de los tutores del centro de práctica. Fuente: elaboración propia a partir del análisis de la información. 
en que esta experiencia formativa se caracteriza por una serie de aportes que consolidan competencias genéricas tales como la comunicación, el trabajo en equipo, la autoconfianza y el desarrollo de niveles de competencias referidas al área disciplinar: "La práctica contribuye con los estudiantes a mejorar en la contabilidad, aprenden contenidos más actualizados en relación a las Normas Financieras Internacionales" (Tutor 21, comunicación personal, 29 de abril de 2016).

Con respecto a las opiniones de los tutores sobre el desempeño de los estudiantes (véase la Tabla 2), se logró identificar que, en general, los tutores perciben que hay competencia para el trabajo en las organizaciones, sobre todo, a nivel de compromiso con la tarea $(\mu=5,9)$, y de la comunicación oral y escrita $(\boldsymbol{\mu}=\mathbf{5}, 8)$.

\section{Una visión de conjunto desde la mirada de los actores: construir puentes entre la teoría y la práctica}

La formación del educador comercial, hoy en día, requiere de experiencias de aprendizaje que vinculen los aspectos teóricos y prácticos. Recalcamos que, desde la visión conjunta obtenida mediante la triangulación de la narrativa de los tres actores de este estudio, las experiencias de aprendizaje situado en ambientes profesionales del área comercial favorecen la consolidación de conocimientos teóricos, el desempeño profesional, la movilización de competencias de alto nivel y el desarrollo de habilidades blandas.

De ahí que los tres grupos de participantes consideran esta experiencia formativa un puente entre el mundo universitario y el de las organizaciones; para ellos es un camino de doble vía, en el cual los docentes asesores reciben retroalimentación de los tutores de los centros de práctica sobre aquellas áreas del saber que se requiere fortalecer en la universidad, y los centros de práctica se benefician de los saberes académicos que ponen en práctica los estudiantes. De esta forma, ambas instituciones cooperan con la formación de un profesional de la docencia que potencia el mundo del trabajo, la inclusión financiera, la cooperación y el emprendedurismo como vías del desarrollo humano sostenible. Además, los actores coinciden en valorar la práctica como un espacio de gran provecho para el desarrollo de competencias que sirven en la vida, como, por ejemplo, el trabajo en equipo, la autoconfianza y la comunicación efectiva.

Tabla 2. Opiniones de los tutores sobre el desempeño profesional del practicante

\begin{tabular}{|c|c|c|c|c|c|c|c|c|c|c|c|c|c|c|}
\hline \multirow{2}{*}{ Opiniones } & \multirow{2}{*}{$\boldsymbol{\mu}$} & \multirow{2}{*}{$\sigma$} & \multicolumn{2}{|c|}{1} & \multicolumn{2}{|c|}{2} & \multicolumn{2}{|c|}{3} & \multicolumn{2}{|c|}{4} & \multicolumn{2}{|c|}{5} & \multicolumn{2}{|c|}{6} \\
\hline & & & f & $\%$ & $f$ & $\%$ & $f$ & $\%$ & $f$ & $\%$ & $f$ & $\%$ & $f$ & $\%$ \\
\hline $\begin{array}{l}\text { Capacidad para comunicarse } \\
\text { de manera efectiva a través del } \\
\text { lenguaje oral y escrito. }\end{array}$ & 5,8 & 0,47 & - & - & - & - & - & - & 1 & 3,0 & 4 & 12,1 & 28 & 84,8 \\
\hline $\begin{array}{l}\text { Capacidad para identificar } \\
\text { problemas, planificar } \\
\text { estrategias y enfrentarlos. }\end{array}$ & 5,6 & 0,66 & - & - & - & - & - & - & 3 & 9,1 & 8 & 24,2 & 22 & 66,7 \\
\hline $\begin{array}{l}\text { Capacidad para participar en } \\
\text { proyectos grupales. }\end{array}$ & 5,7 & 0,59 & - & - & - & - & - & - & 2 & 6,1 & 6 & 18,2 & 25 & 75,8 \\
\hline $\begin{array}{l}\text { Capacidad para dominar el } \\
\text { lenguaje informático, necesario } \\
\text { en el ejercicio de la profesión. }\end{array}$ & 5,7 & 0,65 & - & - & - & - & 1 & 3,0 & - & - & 8 & 24,2 & 24 & 72,7 \\
\hline $\begin{array}{l}\text { Demuestra compromiso en } \\
\text { el desarrollo de las funciones } \\
\text { asignadas. }\end{array}$ & 5,9 & 0,17 & - & - & - & - & - & - & - & - & 1 & 3,0 & 32 & 97,0 \\
\hline
\end{tabular}

Fuente: elaboración propia. 
Pese a los beneficios que las experiencias de práctica aportan a la formación, los tres grupos de participantes del estudio señalan una serie de dificultades que deben afrontarse durante su ejecución. Por tanto, consideramos que a fin de consolidar la práctica como un espacio de aprendizaje complejo se requieren algunos elementos clave (véase la Figura 4).

- Reconocer el perfil de los estudiantes, sus condiciones económicas, sociales, familiares y académicas, de tal manera que se realicen las adaptaciones necesarias y que estas condiciones, lejos de excluir a los estudiantes, se conviertan en áreas de oportunidad.

- Fortalecer los mecanismos y los dispositivos que consoliden los procesos de asesoramiento y feedback. El hecho de que los estudiantes realicen su formación desde la modalidad a distancia trae consigo la necesidad de potenciar estos procesos. En ese sentido, las TIC juegan un papel fundamental para concretar estas importantes estrategias en los procesos de vinculación entre la universidad y el contexto profesional.
- Consolidar los espacios de asesoría con procesos reflexivos y críticos del desempeño de los estudiantes. Por tanto, el docente asesor debe propiciar las condiciones para que los futuros profesores reflexionen sobre las dimensiones cognitivas, afectivas y procedimentales que entran en juego en los procesos de práctica (Korthagen, 2010).

- Establecer fuertes lazos con los centros de práctica a través del trabajo de los tutores, quienes contribuyen con la adaptación del estudiante al centro y con la integración a la cotidianidad del desempeño profesional. Asimismo, se espera que el tutor promueva la autoconfianza y brinde feedback sobre las áreas que debe potenciar (Freixa et al., 2012).

\section{Discusión}

La formación inicial de docentes durante los últimos años ha cobrado un papel preponderante en las políticas de Estado. El foco de la discusión pone en relieve los modelos formativos que impulsan los

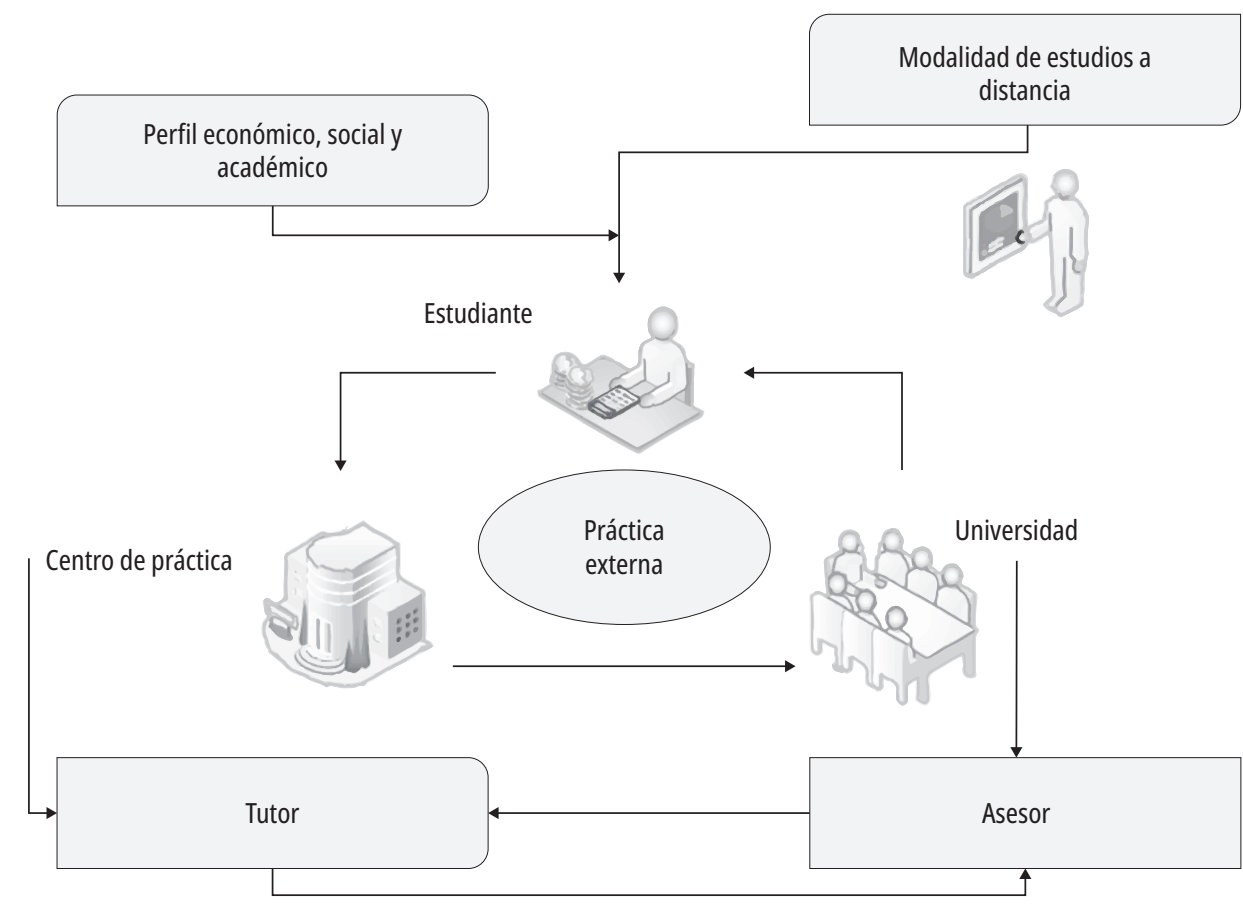

Figura 4. Elementos claves para el desarrollo de las prácticas externas. Fuente: elaboración propia a partir del análisis relacional de las categorías del estudio. 
espacios de práctica profesional como un medio para mejorar la preparación de los profesionales de la docencia (López, 2014). Nuestros datos revelan que el contacto de los estudiantes con el campo laboral les permite el desarrollo de competencias blandas, como, por ejemplo, la comunicación asertiva, la autoconfianza y el trabajo en equipo. Este hecho concuerda con lo expuesto por Vizenor et al. (2017), quienes indican que las prácticas externas ofrecen un espacio sólido y permanente para el desarrollo integral de los estudiantes universitarios.

La investigación desarrollada en este campo ha aportado evidencias que concuerdan con los resultados de este proyecto, e identifican que las experiencias de práctica externa centradas en el fortalecimiento de competencias disciplinares favorecen el desarrollo de habilidades y actitudes necesarias en el campo laboral de las finanzas, la contabilidad y la administración (Ibrahim, 2011; Isah, 2003; Tejada-Fernández et al., 2017; Uramah, 2003). Para crear este entorno en una modalidad de educación a distancia será importante diseñar un sistema de acompañamiento permanente a los estudiantes, mediado por una plataforma virtual $y$ otros recursos que generen una comunicación activa, y a la vez permitan la retroalimentación constante por parte del docente asesor de práctica, ya que, a diferencia de la modalidad presencial, los encuentros de acompañamiento tutorial se realizan con poca frecuencia o con intervalos de tiempo más aislados.

Desde la mirada de los tutores en los centros de práctica, nuestros hallazgos revelan la necesidad de fortalecer los vínculos con los docentes asesores de la universidad con el propósito de mejorar los procesos de retroalimentación entre las partes. Estos resultados están en línea con los planteamientos de Onnoh y Oluseyi (2017), quienes explican que los espacios de colaboración entre las instituciones de educación superior y las del campo laboral pueden contribuir a la mejora de la capacidad de los educadores empresariales, pues permite la transferencia de conocimiento. Las prácticas ofrecen a los estudiantes un espacio para "aprender haciendo y en contexto", lo que favorece la creación de una gama de experiencias que utilizan los docentes en preservicio durante el desarrollo de la práctica profesional en el campo de la docencia. A su vez, las experiencias previas derivadas de la condición de estudiantes-trabajadores, en una modalidad de educación a distancia, les permite establecer aprendizajes más profundos. Este tipo de aprendizaje se caracteriza por el logro de procesos metacognitivos, la autonomía y el aprendizaje para toda la vida (Huberman, Bitter, Anthony y O’Day, 2014).

Si bien es cierto que las prácticas externas son de gran provecho para la formación inicial de docentes, es preciso recalcar que en el caso de los participantes de este estudio se identifican dificultades para su óptima ejecución, como, por ejemplo, las de tipo económico, el poco conocimiento y la falta de manejo de software contable y Excel, así como la gestión del tiempo, debido a que son estudiantes que trabajan y han optado por una modalidad de estudios a distancia. Estas y otras condiciones de los estudiantes se atienden desde la asesoría docente y el acompañamiento de los tutores. Ambos procesos de mediación pedagógica se consideran un elemento de real importancia para la formación de los estudiantes, en especial durante el proceso de práctica.

Los docentes asesores coinciden en que la inducción apropiada al centro de práctica, la asesoría y la supervisión son factores determinantes en la ejecución de esta experiencia, ya que generan un proceso sustentado en el modelamiento académico y en la atención a las diferencias individuales. De igual forma, se visualiza la importancia de establecer un equilibrio a la hora de gestionar el tiempo, puesto que la condición de los estudiantes de educación a distancia representa un desafío al momento de efectuar el proceso de la práctica.

La implementación de la Práctica Administrativa Contable desde la perspectiva de los asesores permite un espacio que aporta al estudiante niveles complejos de desempeño profesional acordes con el estado del arte del campo disciplinar. Moreira et al. (2015) concuerdan con esta perspectiva al argumentar que las instituciones de educación superior deben impulsar la vinculación entre la teoría y la práctica, entre la academia y el mundo laboral. 


\section{Conclusiones}

Este artículo estudia las percepciones de los actores involucrados en el proceso de prácticas externas de los estudiantes de Educación Comercial de la modalidad a distancia, como una experiencia de vinculación entre teoría y práctica. De acuerdo con nuestros resultados, concluimos que la preparación inicial de docentes requiere de espacios curriculares en los planes de estudio que promuevan experiencias de aprendizaje situado, así como la creación de puentes entre los saberes teóricos y los prácticos. Dentro de esa gama de posibilidades se ha identificado que las prácticas externas orientadas a favorecer las competencias disciplinares y las blandas derivan en un proceso formativo integral y de aprendizajes profundo, gracias al espacio profesional que ofrecen los centros de práctica. Consideramos que el éxito de este tipo de experiencias formativas radica en la integración de los centros de práctica y las instituciones formadoras de docentes: esta relación debe caracterizarse por ser estrecha, dialógica y en un permanente intercambio de saberes.

Sin embargo, a fin de que las prácticas sean significativas para los estudiantes se deben tomar en consideración sus características sociales, económicas y académicas, ya que nuestros hallazgos revelan que factores familiares, laborales y de saberes conceptuales y procedimentales constituyen las principales limitaciones en el desarrollo de estas prácticas. El reconocimiento de estas diferencias debe ser la base para el desarrollo de los procesos de asesoría, feedback y seguimiento al progreso de los practicantes.

A la luz de nuestra revisión teórica y del estudio empírico identificamos cómo a los estudiantes el proceso de práctica les ofrece un entorno privilegiado para la consolidación de sus saberes disciplinares, debido a que les otorga un espacio que les permite contrastar y aplicar los aprendizajes teóricos. Además, las prácticas promueven aspectos que favorecen el desarrollo profesional de los docentes en formación tales como la autoconciencia, la reflexión guiada y el intercambio de experiencias.
Por su parte, los asesores y tutores valoran satisfactoriamente este tipo de estrategias, ya que se convierten en un espacio de formación integral y de vínculo entre las instituciones académicas de educación superior, el contexto social y las organizaciones.

Asimismo, concluimos que la práctica cumple una doble función al convertirse en un dispositivo de vinculación entre el saber teórico y el práctico, y en un entorno de aprendizaje que permite consolidar el saber disciplinar como aspecto fundamental para una buena docencia.

Pensamos que futuras investigaciones pueden orientarse a valorar cómo estos dispositivos y estrategias contribuyen al desarrollo de competencias docentes, o bien indagar sobre las estrategias más efectivas para favorecer los vínculos entre la universidad y los centros de práctica.

En fin, consideramos necesario el uso de técnicas de observación como herramientas de recogida de información, puesto que de esta forma es posible efectuar una comparación constante entre las diversas fuentes de la investigación. En tal sentido sugerimos la incorporación de este tipo de técnicas en una futura investigación cuyo foco sea el desarrollo de prácticas en la formación inicial de docentes.

\section{Referencias}

Abarca, A., Alpízar, F., Rojas, C. \& Sibaja, G. (2012). Técnicas cualitativas de investigación. San José : Editorial UCR.

Almeyda-Hidalgo, L. (2016). Arrojados en la acción: aprender a enseñar en la experiencia de práctica profesional. Estudios Pedagógicos (Valdivia), 42(4), 11-30. doi: https://doi.org/10.4067/S0718-07052016000500002

Álvarez, C. \& Hevia, I. (2013). Posibilidades y límites de la relación teoría-práctica en la formación inicial del profesorado. Cultura y Educación, 25(3), 337-346. doi: https://doi.org/10.1174/113564013807749759

Araya-Muñoz, I. \& España-Chavarría, C. (2012). La autonomía profesional. Competencia clave para el docente de educación comercial. Educare, 16(2), 163-170. Recuperado de: http://www.revistas.una.ac.cr/index.php/ EDUCARE/article/view/3937 
Badali, S. (2008). Student teacher stress in the extended practicum: a Canadian context. Policy and Practice in Education, 14, 10-36. Recuperado de: https://ourspace. uregina.ca/bitstream/handle/10294/601/PPE_14_1-2. pdf? sequence $=4 \&$ is Allowed $=y$

Berliner, D. C. (2000). A personal response to those who bash teacher education. Journal of Teacher Education, 51(5), 358-371. doi: https://doi. org/10.1177/0022487100051005004

Caires, S., Almeida, L. S. \& Martins, C. (2010). The socioemotional experiences of student teachers during practicum: A case of reality shock? The Journal of Educational Research, 103(1), 17-27.doi: https://doi. org/10.1080/00220670903228611

Canales-García, A. \& Araya-Muñoz, I. (2017). Teaching resources for commercial education learning: a higher education learning experience systematization. Revista Electrónica Educare, 21(2), 1-23. doi: https://doi. org/10.15359/ree.21-2.7

Chaves-Torres, A. (2017). La educación a distancia como respuesta a las necesidades educativas del siglo XxI. Academia y Virtualidad, 10(1), 23-41. doi: https://doi. org/10.18359/ravi.2241

Choy, D., Wong, A. F., Goh, K. C. \& Ling Low, E. (2014). Practicum experience: pre-service teachers' self-perception of their professional growth. Innovations in Education and Teaching International, 51(5), 472-482. doi: https://doi.org/10.1080/14703297.2013.791552

Cifuentes, G. (2011). Diseño de proyectos de investigación cualitativa. Argentina: Noveduc.

Clayton, K., Smith, H. \& Dyment, J. (2014). Pedagogical approaches to exploring theory-practice relationships in an outdoor education teacher education program. Asia-PacificJournalofTeacherEducation, 42(2), 167-185. doi: https://doi.org/10.1080/1359866X.2014.894494

Crew, T. \& Bodenhamer, J. (2009). Preparing student teaching interns: advice form current business educators. The Delta Pi Epsilon Journal, 51(1), 43-55. Recuperado de http://web.a.ebscohost.com/ehost/pdfviewer/pdfviewer?vid=1\&sid=b7ee8231-899b-4402-88db-224453 80 eec0\%40sdc-v-sessmgr01

Darling-Hammond, L. \& Bransford, J. (2007). Preparing teachers for a changing world: what teachers should learn and be able to do. San Francisco, CA: Jossey-Bass.

Feigenblatt, O. F. von. (2015). Integración de teoría y práctica en educación comercial. Revista de Comunicación de la SEECI, 19(35), 172-176. doi: http://dx.doi. org/10.15198/seeci.2015.36.178-187
Fletcher, E. C., Mountjoy, K. \& Bailey, G. (2011). Exploring the preparedness of business education teacher candidates for their internships: the perspectives of mentor teachers. International Journal of Adult Vocational Education and Technology, 2(4), 28-42. doi: https://doi. org/10.4018/javet.2011100103

Freixa, M., Novella, A. \& Pérez, N. (2012). Elementos para una buena experiencia de prácticas externas que favorece el aprendizaje. Barcelona: Editorial Octaedro.

George, D. \& Mallery, P. (2003). spss for Windows step by step: a simple guide and reference. 11.0 update ( $4^{\mathrm{a}}$ ed.). Boston: Allyn \& Bacon.

Gonzáles, L. \& Larraín, A. (2005). Formación universitaria basada en competencias: aspectos referenciales. En Currículo Universitario Basado en Competencias (pp. 24-57). Barranquilla, Colombia: Cinda. Recuperado de https://docplayer.es/2160507-Curriculo-universitario-basado-en-competencias.html

Gray, C., Wright, P. \& Pascoe, R. (2017). Raising the curtain: investigating the practicum experiences of pre-service drama teachers. Australian Journal of Teacher Education, 42(1), 36-53. doi: https://doi. org/10.14221/ajte.2017v42n1.3

Grossman, P., Schoenfeld, A. \& Lee, C. (2005). Teaching subject matter. En L. Darling-Hammond \& J. Bransford (Eds.), Preparing teachers for a changing world: what teachers should learn and be able to do (pp. 201231). San Francisco, CA: John Wiley \& Sons, Inc.

Grosu, C., Almasan, A. \& Circa, C. (2015). Difficulties in the accounting research-practice teaching relationship: evidence from Romania. Accounting and Management Information Systems, 14(2), 275-302. Recuperado de: http://www.cig.ase.ro/articles/14_2_3.pdf

Grudnoff, L. (2011). Rethinking the practicum: limitations and possibilities. Asia-Pacific Journal of Teacher Education, 39(3), 223-234. doi: https://doi.org/10.1080/1359866X.2011.588308

Huberman, M., Bitter, C., Anthony, J. \& O’Day, J. (2014). The shape of deeper learning: strategies, structures, and cultures in deeper learning network high schools. Findings from the study of deeper learning opportunities and outcomes: Report 1. American Institutes for Research. Recuperado de https://files.eric.ed.gov/fulltext/ED553360.pdf

Ibrahim, M. Y. (2011). Relevance of students' industrial work experience scheme to business education programme in colleges of education in Nigeria (MEd project submitted to the Department of vocational and Tech- 
nical Education, Faculty of Education). Ahmadu Bello University, Zaria.

Isah, A. R. (2003). The effect of students' industrial work experience scheme on skill development of business education students (a case study of Federal College of Education, Kano) (a project submitted to Department of Vocational and Technical Education). Ahmadu Bello University, Zaria.

Kane, M., Berryman, S., Goslin, D. \& Meltzer, A. (1990). Indentiffying and describing the skills required by work. Washington: The Secretarys Commision on Achieving Necessary Skills.

Korthagen, F. A. \& Kessels, J. P. (2009). Linking theory and practice: changing the pedagogy of teacher education. Educational Researcher, 28(4), 4-17. doi: https://doi.or$\mathrm{g} / 10.3102 / 0013189 \mathrm{X} 028004004$

Korthagen, F. (2010). La práctica, la teoría en la formación del profesorado. Revista Interuniversitaria de Formación del Profesorado, 2, 83-101. Recuperado de http://dare.ubvu.vu.nl/bitstream/ handle/1871/22807/247824.pdf?sequence=2

Kug, G. (2008). High-impact educational practices: what they are, who has acces to them, and why they matter. Washington: Association of American Colleges and Universities.

Lawshe, C. H. (1975). A quantitative approach to content validity 1. Personnel Psychology, 28(4), 563-575. doi: https://doi.org/doi:10.1111/j.1744-6570.1975.tb01393

Linuesa, M. C. (2007). La complejidad de las relaciones teoría-práctica en educación. Teoría de la educación. Revista Interuniversitaria, 19(1), 25-46. Recuperado de: http://revistas.usal.es/ revistas_trabajo/index. php/1130-3743/article/view/3235/3260

Loibl, C. \& Fisher, P. J. (2013). Academic discipline and personal finance instruction in high school. Journal of Financial Counseling and Planning, 24(1), 15-33. Recuperado de: https://files.eric.ed.gov/fulltext/EJ1043220. pdf

López, F. (2014). Fortalecer la profesión docente: un desafío crucial. Madrid: Narcea.

Miles, M. \& Huberman, A. (1984). Qualitative data analysis: a source book of new methods. Beverly Hills: Sage.

Moreira, J., Vieira, M. das G. \& da Silva, C. G. (2015). Among theory, practice and technology: the relation between theoretical and practical knowledge in the context of accounting training and the thinking of Jürgen Habermas. Brazilian Business Review, 12(4), 123-139. doi: https://doi.org/10.15728/bbr.2015.12.4.6
National Association for Business Teacher Education. (2010). Business teacher education curriculum guide and program standards. Reston, vA: National Business Education Association.

Onnoh, G. \& Oluseyi, D. (2017). Promoting institutional collaborations for capacity improvement of business educators in tertiary institutions in Delta State of Nigeria. Journal of Education and Practice, 8(14), 7987. Recuperado de https://files.eric.ed.gov/fulltext/ EJ1143858.pdf

Polkinghorne, F. (2015). A content analysis: coursework for the effective preparation of business education teachers. The Journal of Research in Business Education, 57(1), 1-18.

Pozo, R. M. del, Fernández-Lozano, P., González-Ballesteros, M. \& Juanas, A. de. (2013). El dominio de los contenidos escolares: competencia profesional y formación inicial de maestros. Revista de Educación, 1-18. Recuperado de https://www.researchgate.net/profile/ Angel_De_Juanas_Oliva/publication/235724038_ Mastery_of_Content_Professional_Competence_and_Pre-service_Teacher_Education/ links/02bfe512e3f165773c000000.pdf

Ralf, E., Walker, K. \& Wimmer, R. (2008). The pre-service practicum: perspectives of students from three disciplines. Higher Education Perspectives, 4(1), 1-20.

Rozada, J. M. (2007), ¿Son posibles los puentes entre la teoría y la práctica por todo el mundo demandados, sin pilares intermedios?, En J. Romero y A. Luis (coord.), La formación del profesorado a la luz de una profesionalidad democrática (pp. 47-53). Santander: Consejería de Educación de Cantabria.

Tejada-Fernández, J., Carvalho-Dias, M. L. \& Ruiz-Bueno, C. (2017). El prácticum en la formación de maestros: percepciones de los protagonistas. Magis, Revista Internacional de Investigación en Educación, 9(19), 91114. doi: 10.11144/Javeriana.m9-19.pfmp

Tenti, E. (2007). La condición docente: análisis comparado de la Argentina, Brasil, Perú y Uruguay. Argentina: Siglo XxI.

Tilson, J., Sandretto, S. \& Pratt, K. (2017). Connecting theory to practice: using preservice teachers' beliefs, theories and video-recorded teaching to prompt a cycle of praxis. Teaching and Teacher Education, 67(1), 454-463. doi: https://doi.org/10.1016/j.tate.2017.07.012

Uramah, M. (2003). The impact of students' industrial work experience scheme on business education students (a thesis submitted to Department of Vocational and Technical Education, Faculty of Education). Ahmadu Bello University, Zaria. 
Vaillant, D. \& Marcelo, C. (2015). El ABC y D de la formación docente. Madrid: Narcea.

Vizenor, N., Souza, T. \& Ertmer, J. J. (2017). Beneficits or participating in service learning business-related classes: assessing the impact on the community partners. The Journal of Reasearch in Business Education, 58(1), 1-15. Recuperado de: https://scholarworks.boisestate. edu/cgi/viewcontent.cgi article $=1009 \&$ context $=$ ctl_ teaching
Yin, R. K. (2013). Validity and generalization in future case study evaluations. Evaluation, 19(3), 321-332. doi: https://doi.org/10.1177/1356389013497081

Zapata-Callejas, J. (2015). El modelo y enfoque de formación por competencias en la educación superior: apuntes sobre sus fortalezas y debilidades. Academia $y$ Virtualidad, 8(2), 24-33. doi: https://doi.org/10.18359/ ravi. 1420 